01.5

\title{
Нейроподобная динамика в системе фазовой автоподстройки частоты с запаздывающей обратной связью
}

\author{
() И.В. Сысоев ${ }^{1,2}$, М.В. Сысоева ${ }^{1,3}$, В.И. Пономаренко ${ }^{1,2}$, М.Д. Прохоров ${ }^{1}$ \\ ${ }^{1}$ Саратовский филиал Института радиотехники и электроники им. В.А. Котельникова РАН, Саратов, Россия \\ ${ }^{2}$ Саратовский национальный исследовательский государственный университет им. Н.Г. Чернышевского, Саратов, Россия \\ ${ }^{3}$ Саратовский государственный технический университет им. Гагарина Ю.А., Саратов, Россия \\ E-mail: ivssci@gmail.com
}

Поступило в Редакцию 27 февраля 2020 г.

В окончательной редакции 16 апреля 2020г.

Принято к публикации 16 апреля 2020г.

На основе системы фазовой автоподстройки частоты, имеющей запаздывающую обратную связь, предложена модель нейроподобной динамики. Модель способна демонстрировать хаотические колебательные режимы, в которых имеются характерные для нейронной активности переключения между качественно различными видами колебаний.

Ключевые слова: система фазовой автоподстройки частоты, запаздывающая обратная связь, нейроподобная динамика, перемежаемость.

DOI: 10.21883/PJTF.2020.14.49665.18267

Задача построения и исследования математических моделей, описывающих динамику нейронов, давно привлекает к себе большое внимание [1]. Наиболее известными динамическими моделями нейронной активности являются модели Ходжкина-Хаксли, ФитцХью-Нагумо, Моррис-Лекара и Хиндмарша-Роуза, описываемые обыкновенными дифференциальными уравнениями, и модели Ижикевича, Рулькова и Курбажа-Некоркина, описываемые точечными отображениями. Подробный обзор этих моделей представлен в [2]. Достоинством многих моделей с непрерывным временем являются биологическая адекватность и наличие соответствия модельных и физиологических параметров. Однако такие модели сложнее исследовать, чем модели с дискретным временем, особенно при моделировании больших ансамблей связанных нейронов. Кроме того, модели с непрерывным временем, как правило, способны воспроизвести генерацию нейронами либо только спайков (отдельных импульсов), либо только берстов (групп из двух или более спайков, идущих подряд друг за другом и перемежаемых периодами отсутствия активности).

Лишенная этого недостатка модель генерации спайков и берстов в непрерывном времени была предложена в [3] на основе системы фазовой автоподстройки частоты [4]. При изменении параметров такая модель может генерировать как одиночные спайки, так и берсты, в том числе с разным количеством спайков в соседних берстах. Однако модель [3] не способна генерировать сложные режимы, в которых имеются переключения между различными видами нейронной активности. Вместе с тем хаотические спайк-берстовые колебания являются типичными для реальных нейронов, а режимы, в которых относительно регулярные колебания мембранного потенциала нейронов перемежаются с нерегулярными, характерны при эпилепсии [5].

В настоящей работе предложена модификация модели нейронной активности $[3,6]$, заключающаяся в введении зависимости одной из динамических переменных модели от времени запаздывания. Динамика модифицированной модели описывается следующей системой дифференциальных уравнений с запаздывающим аргументом:

$$
\begin{gathered}
\frac{d \varphi}{d t}=y, \\
\frac{d y}{d t}=z, \\
\varepsilon_{1} \varepsilon_{2} \frac{d z}{d t}=\gamma-\left(\varepsilon_{1}+\varepsilon_{2}\right) z-\left(1+\varepsilon_{1} \cos \varphi\right) y(t-\tau) .
\end{gathered}
$$

В терминах системы фазовой автоподстройки частоты переменные $\varphi$ и $y$ описывают соответственно разность фаз и разность частот подстраиваемого и опорного генератора, переменная $z$ характеризует скорость изменения $y$, параметр $\gamma$ - начальная частотная расстройка генераторов, $\varepsilon_{1}$ и $\varepsilon_{2}-$ параметры инерционности фильтра в цепи управления, $\tau$ - время запаздывания. Применительно к динамике нейрона переменную $y$ можно интерпретировать как мембранный потенциал, $\gamma$ оказывает воздействие, сходное с воздействием внешнего тока в модели Ходжкина-Хаксли, параметры $\varepsilon_{1}$ и $\varepsilon_{2}$ отражают конечную скорость ионного переноса через мембрану и позволяют задавать необходимый динамический режим, a $\tau$ соответствует времени рефрактерности нейрона после генерации потенциала действия.

Системы с запаздыванием широко применяются для моделирования многих биологических объектов и процессов [7-9]. Наличие запаздывания в модельных уравнениях, в том числе в связях между подсистемами [10], 

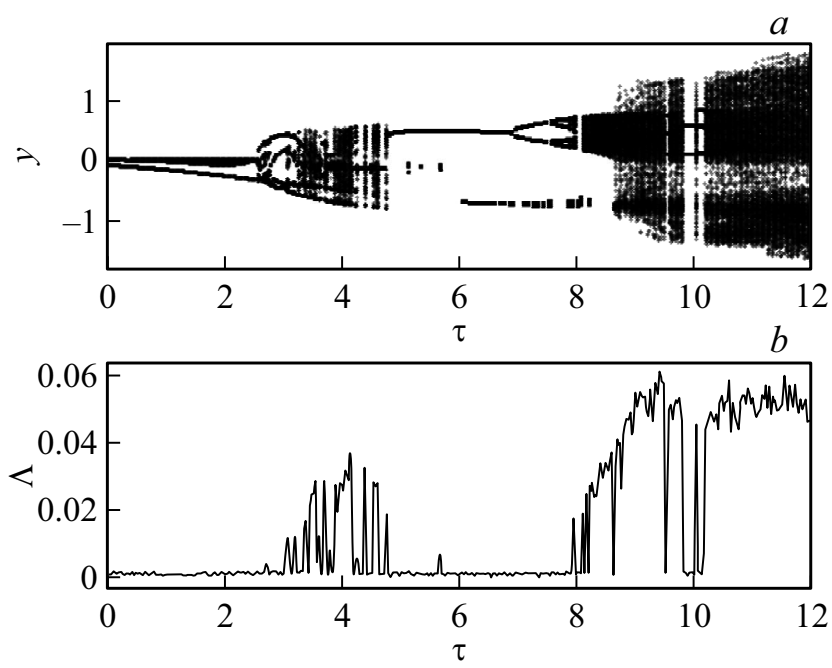

Рис. 1. $a-$ бифуркационная диаграмма зависимости переменной $y$ от времени запаздывания $\tau$ системы (1) при $z=0$, $\gamma=0.075, \varepsilon_{1}=4.5$ и $\varepsilon_{2}=10 . \quad b-$ зависимость старшего показателя Ляпунова системы (1) от времени запаздывания.

позволяет учесть конечную скорость распространения сигналов и развития физиологических процессов. Введение запаздывающей обратной связи в модель способно существенно обогатить ее динамику и увеличить многообразие генерируемых колебательных режимов. Так, система с запаздыванием (1) может демонстрировать большое разнообразие видов нейронной активности, включая спайки, берсты, а также переключения между качественно различными режимами колебаний, которые не наблюдаются в модели при $\tau=0$.

На рис. 1, a построена бифуркационная диаграмма зависимости переменной $y$ от времени запаздывания $\tau$. График построен сечением фазового пространства системы (1) плоскостью $z=0$ при $\gamma=0.075, \varepsilon_{1}=4.5$ и $\varepsilon_{2}=10$. Видно, что с изменением параметра $\tau$ модель демонстрирует как регулярные колебания разного периода, так и нерегулярные колебания. При этом при $\tau<5$ переход к хаосу с ростом $\tau$ происходит через перемежаемость, а при $\tau>6$ переход к хаосу с ростом $\tau$ происходит через каскад бифуркаций удвоений периода. На рис. $1, b$ приведена зависимость старшего показателя Ляпунова $\Lambda$ системы (1) от времени запаздывания при тех же параметрах, что на рис. $1, a$. Для расчета $\Lambda$ мы использовали метод, предложенный в [11] для систем первого порядка с запаздыванием, модифицировав его для системы третьего порядка с запаздыванием. Области положительных значений старшего показателя Ляпунова на рис. $1, b$ хорошо согласуются с областями нерегулярных колебаний на рис. $1, a$, что указывает на хаотический характер нерегулярной динамики.

Исследования системы (1) показывают, что при значениях параметров $\gamma, \varepsilon_{1}$ и $\varepsilon_{2}$, соответствующих при $\tau=0$ периодическим колебаниям, она генерирует хаотические колебания при больших значениях $\tau$. При этом при $\tau \geqslant 10.3$ система ведет себя как классическая система с запаздыванием. Ее динамика определяется преимущественно величиной $\tau$, и система не наследует интересующие нас свойства модели [3], не имеющей запаздывания. В то же время при $\tau \in[3.1,4.8]$ система (1) демонстрирует большой набор колебательных режимов, присущих реальным нейронам.

На рис. 2 приведен временной ряд хаотических колебаний переменной $y$, в котором наблюдаются переключения между относительно регулярными нелинейными колебаниями большой амплитуды (пачки из 11-12 биполярных импульсов) и квазигармоническими колебаниями малой амплитуды. Количество колебаний внутри чередующихся участков ряда с большой и малой амплитудой $y$, а также амплитуды и начальные фазы колебаний на этих участках не являются постоянными.

На рис. 3 изображен временной ряд режима с более сложной перемежаемостью, при которой наблюдаются переключения между тремя различными видами колебаний: 1) почти регулярными колебаниями выше порога возбуждения системы в области $y>0$ с нисходящим трендом среднего; 2) подпороговыми колебаниями с переменной амплитудой; 3) хаотическими спайк-берстовыми колебаниями с небольшим числом импульсов.

Достоинством модельной системы (1) является возможность ее реконструкции по скалярному временно́му ряду только переменной $y$, соответствующей мембранному потенциалу нейрона, измеряемому в эксперименте. При этом переменная $z$ может быть получена численным дифференцированием $y$, а переменная $\varphi-$ численным интегрированием $y$, что частично нивелирует ошибки, связанные с наличием шумов измерений. Для восстановления времени запаздывания по временны́м рядам можно использовать хорошо зарекомендовавшие

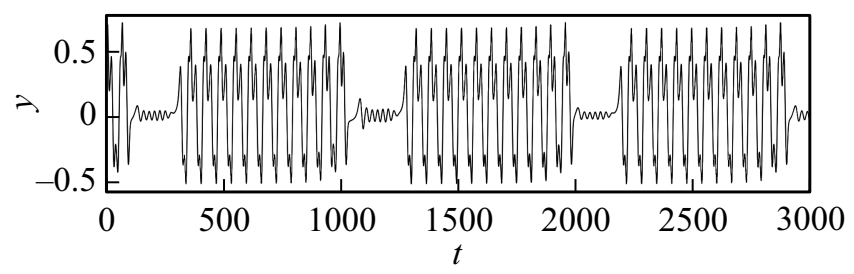

Рис. 2. Временной ряд слабохаотических колебаний переменной $y$ системы (1) при $\tau=3.125, \gamma=0.075, \varepsilon_{1}=4.5$ и $\varepsilon_{2}=10$.

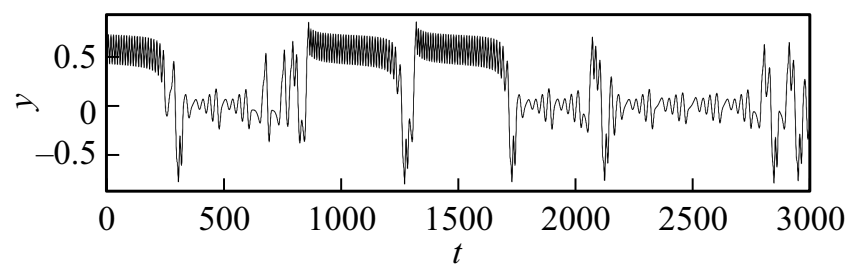

Рис. 3. Временной ряд колебаний переменной у системы (1) в режиме развитого хаоса при $\tau=4.781, \gamma=0.075, \varepsilon_{1}=4.5$ и $\varepsilon_{2}=10$. 
себя методы [12-15]. Кроме того, система (1) может быть реализована в радиофизическом эксперименте с помощью введения запаздывающей обратной связи в систему фазовой автоподстройки частоты, предложенную в [16].

Итак, нами предложена модель нейронной активности на основе системы фазовой автоподстройки частоты, имеющей запаздывание в собственной динамике. Предложенная модель может демонстрировать сложные колебательные режимы, присущие реальным нейронам. В частности, она способна генерировать режимы перемежаемости, для которых характерно наличие сразу нескольких качественно различных типов колебаний при фиксированных значениях параметров и начальных условий системы.

\section{Финансирование работы}

Работа выполнена при поддержке Российского фонда фундаментальных исследований (проект № 19-02-00071).

\section{Конфликт интересов}

Авторы заявляют, что у них нет конфликта интересов.

\section{Список литературы}

[1] Rabinovich M.I., Varona P., Selverston A.I., Abarbanel H.D.I. // Rev. Mod. Phys. 2006. V. 78. P. 1213-1266. DOI: $10.1103 /$ RevModPhys.78.1213

[2] Дмитричев А.С., Касаткин Д.В., Клиньшов В.В., Кириллов С.Ю., Масленников О.В., Щапин Д.С., Некоркин В.И. // Изв. вузов. Прикладная нелинейная динамика. 2018. T. 26. № 4. С. 5-58.

DOI: 10.18500/0869-6632-2018-226-4-5-58

[3] Мищенко М.А., Шалфеев В.Д., Матросов В.В. // Изв. вузов. Прикладная нелинейная динамика. 2012. Т. 20. № 4. C. $122-130$. DOI: $10.18500 / 0869-6632-2012-20-4-122-130$

[4] Шахгильдян В.В., Ляховкин А.А. Системы фазовой автоподстройки частоты. М.: Связь, 1972. $446 \mathrm{c}$.

[5] Lüttjohann A., Pape H.-C. // Sci. Rep. 2019. V. 9. P. 2100. DOI: 10.1038/s41598-018-37985-7

[6] Matrosov V.V., Mishchenko M.A., Shalfeev V.D. // Eur. Phys. J. Spec. Top. 2013. V. 222. P. 2399-2405.

DOI: $10.1140 /$ epjst/e2013-02024-9

[7] Mackey M.C., Glass L. // Science. 1977. V. 197. P. 287-289. DOI: $10.1126 /$ science. 267326

[8] Kuang $Y$. Delay differential equations with applications in population dynamics. Boston: Academic Press, 1993. 398 p. DOI: 10.1016/0378-4754(93)90045-v

[9] Bocharov G.A., Rihan F.A. // J. Comp. Appl. Math. 2000. V. 125. P. 183-199. DOI: 10.1016/S0377-0427(00)00468-4

[10] Kulminskiy D.D., Ponomarenko V.I., Prokhorov M.D., Hramov A.E. // Nonlinear Dynamics. 2019. V. 98. P. 735748. DOI: $10.1007 / \mathrm{s} 11071-019-05224-\mathrm{x}$
[11] Колоскова А.Д., Москаленко О.И., Короновский А.А. // Письма в ЖТФ. 2018. Т. 44. В. 9. С. 19-25. DOI: $10.21883 /$ PJTF.2018.09.46061.17167

[12] Сысоев И.В., Прохоров М.Д., Пономаренко В.И., Безручко Б.П. // ЖТФ. 2014. Т. 84. В. 10. С. 16-26.

[13] Prokhorov M.D., Ponomarenko V.I., Khorev V.S. // Phys. Lett. A. 2013. V. 377. P. 3106-3111. DOI: $10.1016 /$ j.physleta.2013.09.046

[14] Sysoev I.V., Ponomarenko V.I., Kulminskiy D.D., Prokhorov M.D. // Phys. Rev. E. 2016. V. 94. P. 052207. DOI: 10.1103/PhysRevE.94.052207

[15] Сысоев И.В., Пономаренко В.И., Прохоров М.Д. // Изв. вузов. Прикладная нелинейная динамика. 2019. Т. 27. № 4. C. 13-51. DOI: 10.18500/0869-6632-2019-27-4-13-51

[16] Мищенко М.А., Большаков Д.И., Матросов В.В. // Письма в ЖТФ. 2017. Т. 43. В. 13. С. 10-18. DOI: 10.21883/PJTF.2017.13.44806.16737 